\title{
Robot-assisted upper-limb therapy in acute rehabilitation setting following stroke: Department of Veterans Affairs multisite clinical trial
}

\author{
Charles G. Burgar, MD; ${ }^{*}$ Peter S. Lum, PhD; ${ }^{2}$ A. M. Erika Scremin, MD; ${ }^{3}$ Susan L. Garber, MA, OTR; ${ }^{4}$ \\ H. F. Machiel Van der Loos, PhD; ${ }^{5-6}$ Deborah Kenney, MS, OTR; ${ }^{5}$ Peggy Shor, OTR ${ }^{5}$ \\ ${ }^{1}$ Central Texas Veterans Health Care System, Temple, TX, and Department of Mechanical Engineering and Material \\ Sciences, Rice University, Houston, TX; ${ }^{2}$ Washington DC Department of Veterans Affairs (VA) Medical Center, and \\ Department of Biomedical Engineering, Catholic University of America, Washington, DC; ${ }^{3}$ VA Greater Los Angeles \\ Healthcare System, and University of California at Los Angeles, Los Angeles, CA; ${ }^{4}$ Michael E. DeBakey VA Medical \\ Center, and Baylor College of Medicine, Houston, TX; ${ }^{5}$ VA Palo Alto Health Care System, Palo Alto, CA; ${ }^{6}$ Department \\ of Mechanical Engineering, Stanford University, Stanford, CA
}

\begin{abstract}
This randomized, controlled, multisite Department of Veterans Affairs clinical trial assessed robot-assisted (RA) upper-limb therapy with the Mirror Image Movement Enabler (MIME) in the acute stroke rehabilitation setting. Hemiparetic subjects $(n=54)$ received RA therapy using MIME for either up to 15 hours (low-dose) or 30 hours (high-dose) or received up to 15 hours of additional conventional therapy in addition to usual care (control). The primary outcome measure was the FuglMeyer Assessment (FMA). The secondary outcome measures were the Functional Independence Measure (FIM), Wolf Motor Function Test, Motor Power, and Ashworth scores at intake, discharge, and 6-month follow-up. Mean duration of study treatment was 8.6, 15.8, and 9.4 hours for the low-dose, high-dose, and control groups, respectively. Gains in the primary outcome measure were not significantly different between groups at follow-up. Significant correlations were found at discharge between FMA gains and the dose and intensity of RA. Intensity also correlated with FMA gain at 6 months. The high-dose group had greater FIM gains than controls at discharge and greater tone but no difference in FIM changes compared with low-dose subjects at 6 months. As used during acute rehabilitation, motor-control changes at follow-up were no less with MIME than with additional conventional therapy. Intensity of training with MIME was positively correlated with motor-control gains.
\end{abstract}

Clinical Trial Registration: ClinicalTrials.gov, NCT00223808, “Assisted Movement Neuro-rehabilitation: VA Multi-site Clinical Trial”; http://www.clinicaltrials.gov/ct2/show/NCT00223808
Key words: acute, arm, bilateral, dose-response, hemiparesis, intensity, rehabilitation, robotics, stroke, therapy.

\section{INTRODUCTION}

Although the number of deaths from stroke per 100,000 population has decreased almost continuously over the past 50 years [1], stroke continues to be a leading cause of serious, long-term disability [2]. In the

\footnotetext{
Abbreviations: ADL $=$ activity of daily living, ANCOVA = analysis of covariance, ANOVA = analysis of variance, $\mathrm{CG}=$ control group, CIMT = constraint-induced movement therapy, FIM = Functional Independence Measure, FMA = Fugl-Meyer Assessment, MIME = Mirror Image Movement Enabler, NeReBot $=$ Neuro-Rehabilitation-roBot, RA = robot-assisted, Robot-Hi = robot high-dose group, Robot-Lo = robot low-dose group, RR\&DC = Rehabilitation Research and Development Center, SEM = standard error of the mean, VA = Department of Veterans Affairs, WMFT = Wolf Motor Function Test.

* Address all correspondence to Charles G. Burgar, MD; Central Texas Veterans Health Care System, Functional Restoration (007), 1901 Veterans Memorial Drive, Temple, TX 76504; 254-743-0085; fax: 243-742-4966.

Email: Charles.Burgar1@va.gov

DOI:10.1682/JRRD.2010.04.0062
} 
United States, 795,000 new or recurrent strokes occur annually. In 2006, there were 6.4 million stroke survivors [3]. Since 1990, the average length of stay in short-stay hospitals after stroke has decreased by approximately 50 percent for all age groups [1]. Economic pressure to limit the duration of rehabilitative therapies continues to increase. These factors have helped stimulate interest in the use of robot-assisted (RA) techniques to enhance the efficiency and efficacy of poststroke rehabilitation.

The Mirror Image Movement Enabler (MIME) was developed to test a novel method of providing movement therapy that combines bimanual movements with unilateral passive, active-assisted, and resisted movements of the hemiparetic upper limb. Two previous clinical trials demonstrated significantly greater gains in motor recovery [4-8], as measured by proximal upper-limb FuglMeyer Assessment (FMA) of motor function [9] scores, at posttreatment in the RA training group than in the control group (CG). However, in both studies the gains in the RA and CGs were equivalent at the 6-month follow-up evaluations. Our first clinical trial enrolled chronic stroke subjects (>6 months poststroke) and compared the results from equal amounts of RA and conventional therapy delivered during 24 sessions over 8 weeks [4-6]. The second clinical trial enrolled subacute subjects (after discharge from acute rehabilitation, 1 to 5 months poststroke) [7-8]. The subacute training protocol again matched the amount of therapy the RA and CGs received but reduced the dose (used here to indicate the total duration) to 15 one-hour sessions delivered over 4 weeks. Three published reviews of these and other clinical trials have confirmed the potential for RA training to improve proximal upper-limb function; however, functional improvements across studies were not significantly better than in CGs in these analyses [10-12].

Widespread acceptance of robotic systems in the rehabilitation clinic will require demonstration that the therapy delivered using the systems can replace at least a portion of the treatment currently delivered and can do so with added value, such as faster or greater functional recovery, lower cost, or the capability of treating multiple patients simultaneously. In a recent multisite clinical trial involving long-term stroke survivors, Lo et al. found that the cost of high-intensity therapy delivered with human assistance was not significantly different than the cost of RA therapy [13]. Their study confirmed the ability of chronic stroke survivors to benefit from high-intensity, repetitive movements. After 36 weeks, subjects receiving
RA therapy had significantly greater increases in FMA scores than control subjects receiving only usual care. When compared with subjects receiving intensive, therapist-assisted training that was matched in schedule, form, and intensity, the FMA gains were similar in both groups. These results are important because they support the use of robotic systems to deliver effective but very laborintensive therapy. However, a cost advantage was not found. Until an economic advantage is also clear, healthcare payers may resist the financial burden of RA therapy.

The essential characteristics of robotic systems have yet to be identified, creating a barrier to their optimization and economical design. The protocols, timing, limb segments manipulated, and amounts of training have varied widely in clinical trials evaluating RA upper-limb therapy, which may account for the disparate outcome results. To date, no consensus on robotic system design or timing of the training has emerged, possibly because no current system is ideal for treatment of all stroke patients through all clinical stages of recovery. The majority of RA therapy studies have used unilateral training. Previous randomized clinical trials using bimanual devices in the subacute stroke recovery period demonstrated significant motor function gains with similar patterns of recovery (MIME, which facilitates shoulder and elbow movements [7-8], and Bi-Manu-Track, a robotic system targeting forearm- and wrist-movement recovery [14]). The ability of MIME to facilitate motor recovery in subjects with chronic and subacute hemiparesis by using both bimanual and unilateral modes motivated us to evaluate the system in the acute stroke recovery period, when those features may offer advantages over simpler and less costly designs. To evaluate whether MIME could facilitate similar or greater motor recovery as the same amount of early hands-on therapy (the primary aim of this study), we provided training in the acute inpatient rehabilitation setting. A secondary aim was to assess the dose-response effect of RA upper-limb therapy, which has not previously been reported.

\section{METHODS}

\section{Study Design}

Conducting randomized controlled clinical trials in the acute rehabilitation setting poses challenges that influence study design. The current emphasis in acute rehabilitation is on teaching compensatory rather than 
restorative methods because of limits on length of stay and staffing. Until the efficacy of RA therapy is scientifically established and clinically accepted, study-related activities must occur in addition to conventional therapy, which is typically tailored by a treatment team to each patient's functional deficits and tolerance. Conventional treatment often has priority over research interventions in terms of scheduling. Research sessions are likely to be cancelled when they conflict with medical tests and when patients experience fatigue. Previous studies in acute settings had limitations. Aisen et al. [15], Volpe et al. [16] and, more recently, Masiero et al. [17] did not compare RA therapy to equal amounts of conventional therapy, leaving in question whether the robot or the additional therapy resulted in achievement of reported gains. To meet our primary study aim, we assigned subjects in this clinical trial to one of three groups that all received additional therapy targeted at improving motor function of the paretic upper limb.

A prospective, randomized, controlled, multisite clinical trial with outcome raters blinded to group assignment was conducted at the Michael E. DeBakey Department of Veterans Affairs (VA) Medical Center (Houston, Texas), the VA Greater Los Angeles Healthcare System (Los Angeles, California), and the VA Palo Alto Health Care System (Palo Alto, California). Subjects were asked to refrain from discussing study activities with the assessment therapists or other subjects. They were informed that there were three study groups and that all subjects would receive active interventions that differed in dose but not how their group compared with the others. It is possible that subjects did not remain blinded to group assignment, since preventing their learning of another subject's schedule was not practicable when more than one was enrolled at the site.

Before study initiation, training of project staff from all three sites was conducted in person at the Rehabilitation Research and Development Center (RR\&DC) in Palo Alto, California. Training included hands-on robotic system operation and standardization of outcome assessment methods. Enrollment of test subjects in Palo Alto began at that time to allow therapists from the other two sites to observe the assessment and training protocols. All therapists were certified to administer the Functional Independence Measure (FIM) [18]. Additional MIME systems were fabricated at the RR\&DC and then installed at the other sites. The study was monitored and issues were resolved through electronic mail, conference calls, and site visits.

\section{Subjects}

Eligibility for enrollment in accordance with the study inclusion and exclusion criteria was determined for veterans admitted with a primary diagnosis of stroke to the inpatient medical and rehabilitation services at the collaborating sites. Patients with a previous ischemic cerebral event were allowed to participate if they had experienced motor and sensory recovery in the upper limb before the current hospital admission. Patients admitted to a long-term care unit for rehabilitation were allowed to participate if they were receiving at least 2 hours of rehabilitation therapy 5 or more days per week and met other enrollment criteria. Subjects entered the study between 7 and 21 days postictus after receiving medical clearance to participate and giving informed consent. Subjects were excluded if they exhibited upperlimb joint pain that restricted normal movement, had absent proprioception at the elbow or shoulder joints, or scored less than 22 on the Mini-Mental State Examination [19]. Subjects with cardiovascular, orthopedic, or neurological conditions that would have precluded exercise in short-duration, moderate-workload trials were also excluded. Using an assignment list based on a random number table, we first stratified participants by impairment level (initial upper-limb FMA <20, 20-39, $\geq 40$ ) and then assigned them to the robot low-dose group (Robot-Lo), robot high-dose group (Robot-Hi), or CG, which received additional conventional therapy. Subject demographics on entry are shown in Table 1.

\section{Intervention}

All therapy was performed with the supervision of a physical or occupational therapist. The 5-minute preparatory and terminal segments of each session were identical in all three groups. The remainder of each CG session was aimed at improving the function of the paretic upper limb through a variety of therapeutic modalities. The therapy was progressive and tailored to the individual's specific stroke diagnosis, level of impairment, and residual deficits. Treatment interventions addressed edema, loss of flexibility, loss of strength, decreased postural control, abnormal motor activation, and lack of coordination. Specific treatment techniques included soft tissue and joint mobilization at the start of each session, neuromuscular reeducation strategies, isolated progressive resistive 
JRRD, Volume 48, Number 4, 2011

Table 1.

Summary of participant baseline characteristics (mean \pm standard error of the mean, unless otherwise indicated).

\begin{tabular}{lcccc}
\hline \multicolumn{1}{c}{ Characteristic } & Robot-Lo $(\boldsymbol{n}=\mathbf{1 9})$ & Robot-Hi $(\boldsymbol{n}=\mathbf{1 7})$ & Control $(\boldsymbol{n}=\mathbf{1 8})$ & $\boldsymbol{p}$-Value \\
\hline Age (yr) & $62.5 \pm 2.0$ & $58.6 \pm 2.3$ & $68.1 \pm 3.3$ & 0.046 \\
Days Poststroke & $17.3 \pm 2.7$ & $16.6 \pm 2.4$ & $10.6 \pm 1.2$ & 0.08 \\
Side of Stroke (L/R), $n$ & $9 / 10$ & $9 / 8$ & $5 / 13$ & 0.24 \\
Fugl-Meyer (max 66) & $26.7 \pm 5.0$ & $19.0 \pm 3.7$ & $24.2 \pm 4.8$ & 0.49 \\
FIM (upper limb, max 63) & $28.4 \pm 2.6$ & $27.9 \pm 1.7$ & $26.9 \pm 2.0$ & 0.88 \\
Motor Power (max 70) & $27.9 \pm 4.8$ & $21.5 \pm 4.2$ & $24.9 \pm 4.2$ & 0.61 \\
Ashworth (max 5) & $0.44 \pm 0.10$ & $0.31 \pm 0.08$ & $0.33 \pm 0.08$ & 0.49 \\
WMFT FAS (max 5) & $1.6 \pm 0.4$ & $1.0 \pm 0.3$ & $1.1 \pm 0.4$ & 0.50 \\
WMFT MT (s) & $81 \pm 19$ & $85 \pm 17$ & $88 \pm 18$ & 0.87
\end{tabular}

Note: Robot low-dose group (Robot-Lo) received up to 15 hours and robot high-dose group (Robot-Hi) up to 30 hours of robot-assisted upper-limb therapy. Control group received up to 15 extra hours of conventional therapy.

FAS = Functional Ability Scale, FIM = Functional Independence Measure, $\mathrm{L}=$ left, max $=$ maximum score, $\mathrm{MT}=$ movement time, $\mathrm{R}=$ right, $\mathrm{WMFT}=\mathrm{Wolf}$ Motor Function Test.

exercises, and a progression to functional activities of daily living (ADLs) retraining at the same workstation used for the RA sessions. About 5 minutes of each CG treatment session were devoted to exposure to MIME with the robot positioning targets for static and dynamic tracking, reaching, and self range-of-motion tasks. The robot did not apply any forces to the CG subjects during these tasks. This component of the protocol was included to control for potential novelty effects of the robotic intervention.

Both RA groups performed the movements with continuous direct visualization of the limbs, using physical objects as targets to maintain a more functional (using physical instead of virtual targets) and goal-directed set of tasks. Movements progressed from passive, with paretic upper-limb motion controlled by the contralateral limb or by the robot in trajectories predetermined by the therapist, to practice of unilateral active-assisted movements followed by practice of actively resisted movements of the affected limb. As with the CG, subjects in the RA groups were advanced to the more challenging tasks consistent with their level of recovery and ability to complete those movements that required less volitional control and strength. Sessions began with practice of targeted, twodimensional reaching movements at table level and then progressed to more complex, three-dimensional, out-ofsynergy movements beginning at table level and ending at eye level. Movement patterns incorporated into the training sessions have been previously described [6]. Representative beginning and ending arm positions are shown in Figure 1.

Subjects sat in a wheelchair at a height-adjustable table to which the robot and a digitizer were attached.
The height was set to allow unrestricted movement within each subject's functional range of motion during initial assessment and maintained at that height during subsequent sessions. Trunk movement was limited by a contoured seat back with solid shell (Jay Medical, Ltd, Model 182518TK; Longmont, Colorado) and customized

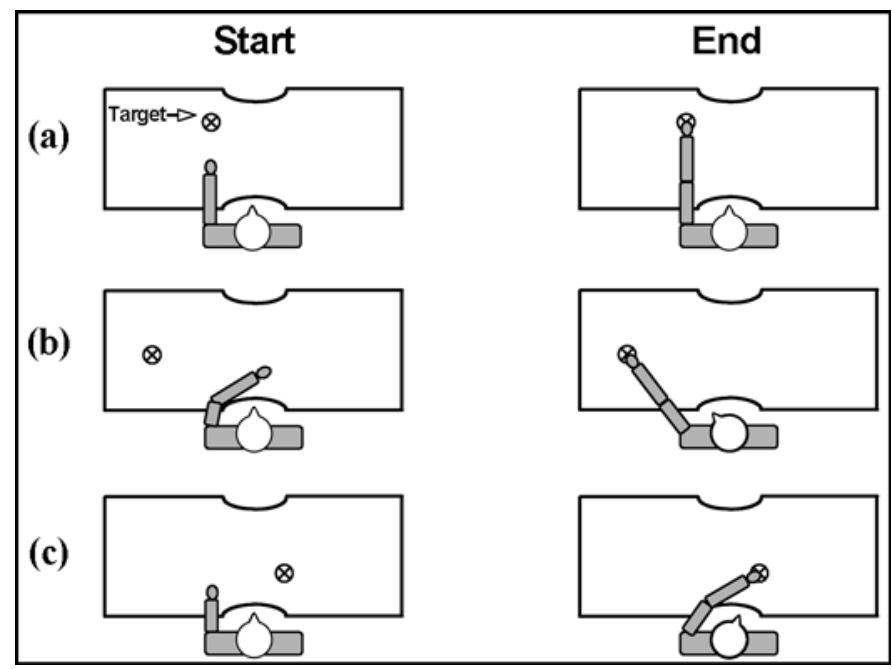

Figure 1.

Examples of unilateral movements practiced with robot assistance. (a) Shoulder flexion, (b) external rotation, and (c) internal rotation can each be practiced in three-dimensional space with increasing degrees of difficulty (e.g., table surface, table-to-shoulder, then tableto-eye levels). Targets are physical items, such as stacked cones, placed on surface of workstation. Each unilateral movement can be practiced in three robot operating modes. Bimanual mode is also available but not illustrated. 
cross-torso straps. The hemiparetic forearm was secured in a splint that stabilized the wrist and hand (Figure 2). A Puma 560 robot manipulator (Unimation, Inc, acquired by Staubli Corp; Duncan, South Carolina-no longer in production) was attached to the splint and applied forces to the limb during training movements. The forces and torques between the robot and the affected limb were measured by a 6-axis sensor (Delta SI 330-30, $0.25 \mathrm{~N}$ resolution, ATI Industrial Automation; Apex, North Carolina). Force and torque applied to the subject's forearm were limited by a pneumatically controlled coupling (QuickSTOP, QS-200NP-T3, Applied Robotics Inc; Glenville, New York) that mechanically decoupled the
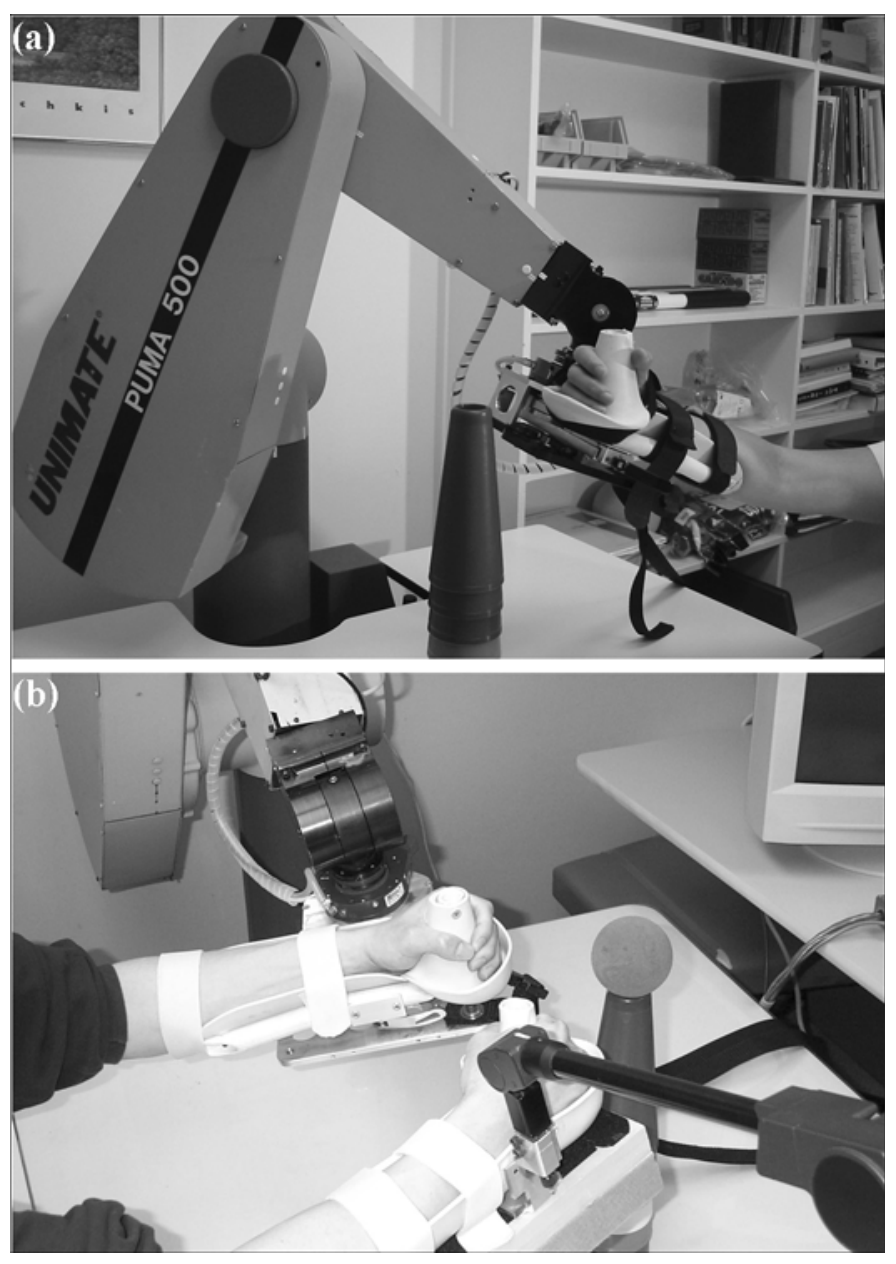

Figure 2.

(a) Unilateral and (b) bimanual modes of training provided by Mirror Image Movement Enabler. subject and stopped the robot whenever interaction torques exceeded a preset level ( $15 \mathrm{~N} \cdot \mathrm{m}$ typically).

The MIME system was programmed to provide four modes of RA training. Forces applied to the subject's forearm assisted or resisted elbow and shoulder movements in three-dimensional space. Three unilateral exercise modes facilitated practice of reaching movements to targets determined for each subject by the therapist, and a bimanual mode enabled subjects to practice mirror-image upper-limb exercises. The unilateral modes were (1) passive mode (the hemiparetic limb was moved to the target by the robot as the subject relaxed initially and then attempted to move with the robot), (2) active-assisted mode (the subject triggered robot movement by applying force in the intended direction of movement and then attempted to move with the robot as it moved the limb to the target), and (3) active-constrained mode (movement only occurred while the subject applied sufficient force to overcome a programmable, velocity-dependent resistance along the desired path, with any off-trajectory force opposed by a stiff springlike load). In the bimanual mode, the subject attempted bilateral mirror-image movements with assistance for the paretic limb provided by the robot as necessary. The contralateral ("normal”) limb was placed in a similar splint and its position and orientation were tracked by a low-inertia, 6 degrees-offreedom digitizer (MicroScribe 3DLX, Immersion, Corp; San Jose, California). This device was used instead of a second Puma robot to avoid inertial effects that could cause undesired sensory input to the less-affected side, simplify system design, increase reliability, and contain costs. The robot continuously moved the paretic limb to the mirror-image position of the opposite limb, as measured by the digitizer, with minimal delay.

The robot was mounted to one side of the table and the digitizer to the other side. We accommodated subjects with right and left hemiparesis by positioning the subject appropriately in the workspace. The digitizer was also used for assessments of voluntary movement kinematics by attaching it to the paretic limb. During RA movements, interaction force and torque measurements from the transducer were recorded and archived by a personal computer. These data and the motions of the robot were monitored by the control program to prevent potentially hazardous situations from occurring. Strategically placed safety switches and mechanical stops were used to deactivate the robot, if necessary. 
Following initial evaluation, we coordinated studyrelated training sessions with those scheduled by the interdisciplinary clinical treatment team. Participation in this study did not affect other rehabilitation therapy or length of hospital stay. Study therapy was terminated when subjects reached the maximum number of sessions planned for their group or when they were discharged from acute inpatient rehabilitation, whichever came first. CG and Robot-Lo subjects were eligible to receive up to 15 one-hour therapy sessions over a 3-week period and Robot-Hi subjects were eligible to receive up to 30 onehour therapy sessions over the same period. Training under the study protocol was as tolerated and in addition to regular physical, occupational, and speech therapy. Subjects' progression from easier to more challenging tasks during study-related therapy was contingent on their meeting performance criteria defined by consensus among therapists from all sites.

\section{Measures}

A second therapist at each site, blinded to group assignment, performed a clinical assessment battery just before study initiation, just after completion of training, and again at the 6-month follow-up. The primary outcome measure was the FMA of the upper limb, which has been shown to be valid and reliable [20-21]. This test consists of 33 items, each rated on a 3-point scale (0-2) for a maximum possible score of 66 . A subset of the test items reflecting proximal upper-limb recovery provided a maximum score of 42 . The FMA assesses isolated voluntary and synergistic movement patterns, grasp, and reflex activity. Several secondary measures were included in the test battery. The strength of 14 shoulder and elbow muscle groups was assessed by performing manual muscle testing of isolated joint actions and applying the Medical Research Council Motor Power grading scale (0-5) with a maximum possible score of 70 (scapular abduction/ upward rotation, scapular elevation, adduction, adduction/ depression, adduction/downward rotation, flexion, extension, abduction, horizontal adduction, horizontal abduction, external rotation, internal rotation, elbow flexion, elbow extension). The upper-limb portion of the FIM $[18,22]$ was assessed (maximum score $=63$ ) to determine treatment impact on independence in ADLs. Although improvement in this measure can occur solely from compensatory techniques, we believe its use as a secondary outcome measure is justified. Since all subjects received the same training to improve ADLs during usual therapy, a group difference in the FIM suggests a treatment effect in the absence of an alternate explanation. Without an eventual clear link between RA therapy and functional improvement, significant improvements in motor control remain largely academic. With the widespread use of the FIM for judging efficiency and effectiveness of rehabilitation programs, excluding it may decrease the credibility given the results by funding agencies and insurers.

Hypertonia was measured in nine muscle groups (shoulder internal rotators, elbow extensors, elbow flexors, pronators, supinators, wrist flexors, wrist extensors, digit flexors, digit extensors) with the Modified Ashworth Scale [23-24]. These nine scores were averaged to produce a composite tone metric. The Wolf Motor Function Test (WMFT) was included as another secondary outcome measure. It consists of six simple and nine complex movement tasks that are timed, each with a limit of 120 seconds [25]. This test has been shown to have high interrater and test-retest reliability and good internal consistency for testing upper-limb function after stroke [26]. Biomechanical measurements of isometric strength and reaching kinematics were also made just before the training, just after the training, and again 6 months later. These data are not reported here.

\section{Data Analysis}

Baseline differences between groups were examined with analysis of variance (ANOVA) followed by Sidak post hoc testing. The WMFT movement time data were transformed with the log function before statistical analysis, because the assignment of 120 seconds to tasks that cannot be completed often produces a nonnormal distribution. Variables that failed the Kolmogorov-Smirnov test of normality were analyzed with the Kruskal-Wallis nonparametric test. Group differences after treatment were tested with analysis of covariance (ANCOVA) on posttreatment scores with pretreatment scores, age, and chronicity as covariates. Between-subjects factors in the ANCOVA were treatment group and testing site. After combining data from Robot-Hi and Robot-Lo subjects to obtain a sample exposed to a range of treatment doses and intensities, we calculated Pearson product moment correlation coefficients for dose versus intake FMA, change in FMA, and change in FIM scores. Correlations were also computed for training intensity versus changes in FMA and FIM scores. 


\section{RESULTS}

A total of 54 subjects completed the immediate posttreatment testing with 5 or more hours of treatment and were retained for analysis. Relocation of some key study team members and start-up issues delayed enrollment of subjects in Los Angeles and Palo Alto by up to 3 months. The breakdown across sites was 23 subjects in Houston, 19 in Los Angeles, and 12 in Palo Alto.

The randomization procedure produced equal proportions of subjects in each group at each test site $(p>0.90)$. There were no differences between sites in the age, duration of treatment provided, or baseline scores on the FMA and FIM $(p>0.10)$. However, the subjects at the Houston site were enrolled earlier after stroke than at the Los Angeles site $(p=0.01)$ and the Palo Alto site $(p=0.056)$. Mean \pm standard error of the mean (SEM) chronicity was $10.4 \pm 1.2,18.5 \pm 2.3$, and $18.1 \pm 3.4$ days in the Houston, Los Angeles, and Palo Alto sites, respectively. The baseline characteristics of these subjects are summarized in Table 1. Group differences in age were significant, with the CG significantly older than the Robot-Hi group ( $p=$ 0.046). The Robot-Hi dose (total hours of study-related therapy) was significantly larger than the other groups $(p<0.005)$, while the dose was not different between the Robot-Lo group and CG $(p>0.90)$. The mean \pm SEM dose was $9.4 \pm 0.9,8.6 \pm 0.7$, and $15.8 \pm 2.2$ hours in the CG, Robot-Lo group, and Robot-Hi group, respectively. Although daily therapy dose goals were established at entrance to the study, few subjects received the maximum allowed for their group. Early discharges, scheduling conflicts, and patient tolerance were among the factors that reduced the total amount of therapy. The difference in dose between the Robot-Hi group and the other groups was due to five subjects who received between 25 and 30 hours of robot therapy (Figure 3). Closer examination of these five Robot-Hi subjects found that they entered the study with lower FMA scores than other subjects in this group. Their mean baseline FMA score was $7.0 \pm 1.6$, compared with $24 \pm 4.5$ for the other subjects ( $p=0.03$ ). A total of 37 subjects returned for the 6-month follow-up evaluations.

Primary and secondary outcome measures are shown in Tables 2 and 3. Recovery profiles for the FMA and upper-limb FIM are plotted in Figures 4 and 5. ANOVA on immediate posttreatment scores found that group differences in the upper-limb FIM were significant $(p=$

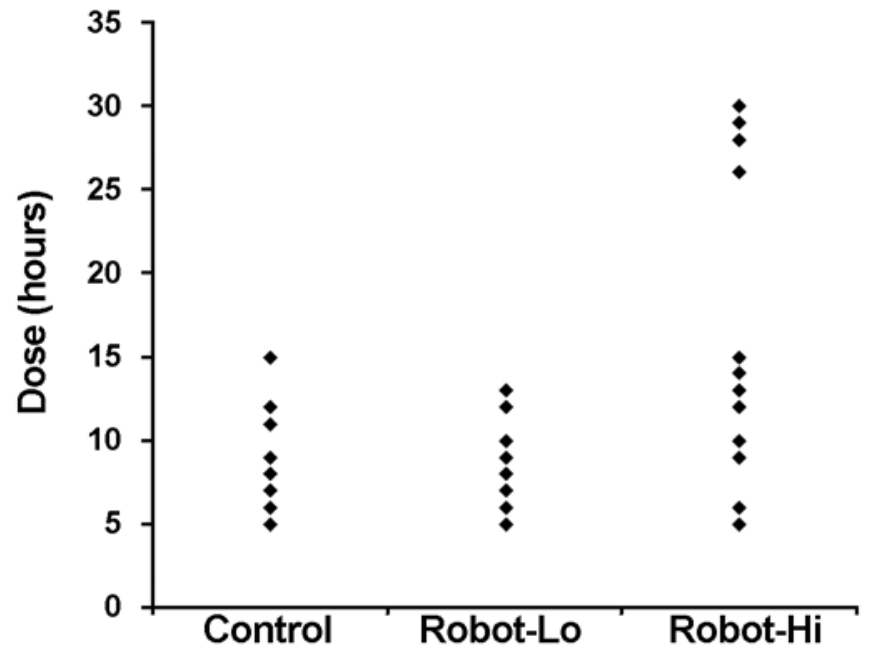

Figure 3.

Distribution of total treatment hours by group. Data points overlap where subjects within each group received same number of hours. Note: Robot low-dose group (Robot-Lo) received up to 15 hours and robot high-dose group (Robot-Hi) up to 30 hours of robot-assisted upper-limb therapy. Control group received up to 15 extra hours of conventional therapy.

0.04). Post hoc comparisons found that this group effect was caused by higher scores in the Robot-Hi group than the CG $(p=0.04)$. Testing site was also a significant factor in the posttreatment FIM score $(p=0.004)$, with the Los Angeles site having higher posttreatment FIM scores than the Houston site $(p=0.003)$. No group differences were found in any of the other outcomes $(p>0.15)$. At the 6-month time point, a significant group difference was detected in the Ashworth test $(p=0.049)$. Post hoc comparisons found that this was due to larger increases in tone in the Robot-Hi group than in the Robot-Lo group $(p=0.04)$. No other group differences were detected at the 6-month follow-up ( $p>0.05)$.

The wide spread in treatment dose provided to the robot-trained subjects motivated an examination of the effect of dose and intensity after collapsing the Robot-Lo and Robot-Hi groups. There was a significant negative correlation between dose and intake FMA ( $r=-0.37, p=$ $0.03)$. Dose was positively correlated with gains in FMA at the immediate posttreatment time point $(r=0.34, p=$ 0.04 ) in the subjects who received robot therapy. However, this effect had weakened to a trend at the 6-month time point ( $r=0.37, p=0.07$ ). Intensity in hours of therapy per day was calculated as dose divided by duration of 
Table 2.

Gains in outcomes at posttreatment (mean \pm standard error of the mean).

\begin{tabular}{lcccc}
\hline \multicolumn{1}{c}{ Outcome Measure } & Robot-Lo $(\boldsymbol{n}=\mathbf{1 9})$ & Robot-Hi $(\boldsymbol{n}=\mathbf{1 7})$ & Control $(\boldsymbol{n}=\mathbf{1 8})$ & $\boldsymbol{p}$-Value \\
\hline Fugl-Meyer (max 66) & $6.8 \pm 1.9$ & $14.4 \pm 3.6$ & $14.0 \pm 3.6$ & 0.47 \\
Fugl-Meyer (proximal, max 42) & $4.2 \pm 0.8$ & $9.1 \pm 2.5$ & $8.8 \pm 2.5$ & 0.48 \\
FIM (upper limb, max 63) & $17.7 \pm 1.9$ & $21.5 \pm 2.1$ & $15.9 \pm 1.5$ & $0.04^{*}$ \\
Motor Power (max 70) & $13.7 \pm 2.3$ & $16.0 \pm 3.0$ & $15.4 \pm 3.7$ & 0.86 \\
Ashworth (max 5) & $0.0 \pm 0.06$ & $0.19 \pm 0.09$ & $0.11 \pm 0.10$ & 0.15 \\
WMFT FAS (max 5) & $0.7 \pm 0.2$ & $0.9 \pm 0.3$ & $1.2 \pm 0.3$ & 0.75 \\
WMFT MT (s) $^{\dagger}$ & $-16.8 \pm 19.0$ & $-29.8 \pm 17.0$ & $-34.4 \pm 18.0$ & 0.65
\end{tabular}

Note: Robot low-dose group (Robot-Lo) received up to 15 hours and robot high-dose group (Robot-Hi) up to 30 hours of robot-assisted upper-limb therapy. Control group received up to 15 extra hours of conventional therapy.

*Robot-Hi > control.

${ }^{\dagger}$ WMFT MT data were transformed with log function before statistical analysis.

FAS $=$ Functional Ability Scale, FIM $=$ Functional Independence Measure, $\max =$ maximum score, MT $=$ movement time, WMFT $=$ Wolf Motor Function Test

Table 3.

Gains in outcomes at 6-month follow-up (mean \pm standard error of the mean).

\begin{tabular}{lcccc}
\hline Outcome Measure & Robot-Lo $(\boldsymbol{n}=\mathbf{1 4})$ & Robot-Hi $(\boldsymbol{n}=\mathbf{1 1})$ & Control $(\boldsymbol{n}=\mathbf{1 2})$ & $\boldsymbol{p}$-Value \\
\hline Fugl-Meyer (max 66) & $15.9 \pm 3.5$ & $23.6 \pm 5.8$ & $15.3 \pm 4.9$ & 0.26 \\
Fugl-Meyer (proximal, max 42) & $10.1 \pm 2.0$ & $14.5 \pm 3.6$ & $8.1 \pm 3.3$ & 0.43 \\
FIM (upper limb, max 63) & $24.2 \pm 2.9$ & $27.5 \pm 3.0$ & $26.8 \pm 3.1$ & 0.90 \\
Motor Power (max 70) & $18.0 \pm 3.3$ & $27.8 \pm 4.0$ & $24.4 \pm 4.8$ & 0.15 \\
Ashworth (max 5) & $0.02 \pm 0.14$ & $0.83 \pm 0.25$ & $0.16 \pm 0.15$ & $0.049^{*}$ \\
WMFT FAS (max 5) & $1.2 \pm 0.4$ & $1.8 \pm 0.7$ & $2.1 \pm 0.5$ & 0.05 \\
WMFT MT (s) $^{\dagger}$ & $-27.4 \pm 13.0$ & $-57.2 \pm 11.0$ & $-45.7 \pm 10.0$ & 0.08 \\
\hline
\end{tabular}

Note: Robot low-dose group (Robot-Lo) received up to 15 hours and robot high-dose group (Robot-Hi) up to 30 hours of robot-assisted upper-limb therapy. Control group received up to 15 extra hours of conventional therapy.

${ }^{*}$ Robot-Hi > Robot-Lo.

${ }^{\dagger}$ WMFT MT data were transformed with log function before statistical analysis.

FAS = Functional Ability Scale, FIM = Functional Independence Measure, max = maximum score, MT = movement time, WMFT = Wolf Motor Function Test.

treatment. The combined robot group had a very strong correlation between intensity and FMA outcome posttreatment $(r=0.45, p=0.005)$ and at 6 months $(r=0.66, p<$ $0.001)$. The CG did not show this effect at posttreatment ( $r=-0.19, p>0.40)$ or at follow-up $(r=-0.56, p>0.05)$. The correlation between intensity and FMA gains in the robot groups was possibly confounded by the fact that subjects who received a higher dose had lower baseline FMA scores and so had more room for improvement. To eliminate this possibility, we performed a hierarchical multiple regression with baseline FMA score entered initially as the sole predictor of FMA gains. Then, intensity was added to the model to determine whether it significantly predicts FMA gain after the effects of baseline FMA score have been accounted for. For FMA gains at the posttreatment time point, FMA score at baseline was not a significant predictor $(p>0.80)$, while intensity was significant $(p=$

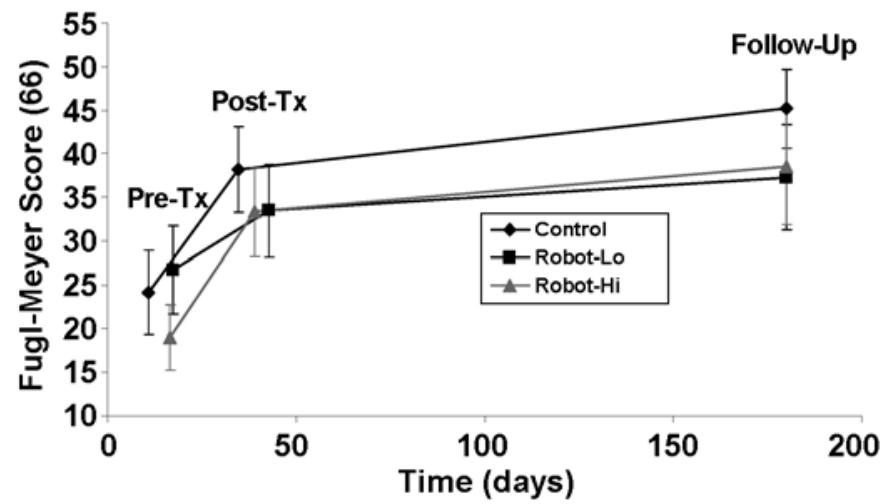

Figure 4.

Scores on Upper-Limb Fugl-Meyer at pretreatment (Pre-Tx), posttreatment (Post-Tx), and 6-month follow-up. Note: Robot low-dose group (Robot-Lo) received up to 15 hours and robot high-dose group (Robot-Hi) up to 30 hours of robot-assisted upper-limb therapy. Control group received up to 15 extra hours of conventional therapy. 


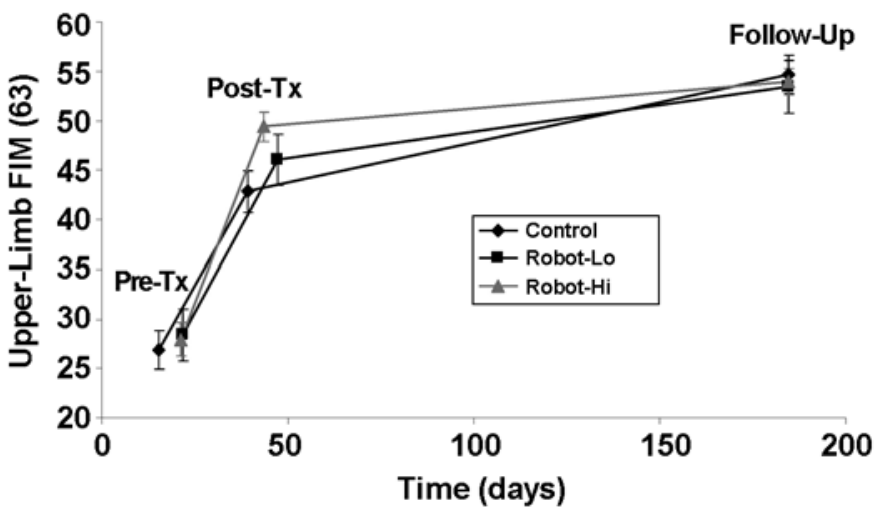

Figure 5.

Scores on upper-limb portion of Functional Independence Measure (FIM) at pretreatment (Pre-Tx), posttreatment (Post-Tx), and 6-month follow-up. Note: Robot low-dose group (Robot-Lo) received up to 15 hours and robot high-dose group (Robot-Hi) up to 30 hours of robot-assisted upper-limb therapy. Control group received up to 15 extra hours of conventional therapy.

0.007). Similarly, at the follow-up time point, baseline FMA score did not predict FMA gain $(p>0.80)$, while intensity was a significant predictor $(p<0.001)$.

The greater increases in Ashworth scores at followup in the Robot-Hi group compared with the Robot-Lo group motivated further examination. We performed correlations between Ashworth score increases at the followup time point and gains in the impairment and function scales (FMA, FIM, WMFT) and found no significance $(p>0.60)$ when all the robot-trained subjects were grouped together. Ashworth score increases in the RobotHi group were distributed throughout the upper limb. At the follow-up time point, mean \pm SEM Ashworth score increases at the finger flexors, wrist flexors, elbow flexors, and shoulder internal rotators were $1.4 \pm 0.5,1.1 \pm$ $0.3,1.2 \pm 0.4$, and $1.0 \pm 0.3$, respectively.

\section{DISCUSSION}

With the exception of age, subjects in the three groups did not differ significantly in baseline characteristics. Robot-Hi subjects were younger on average than controls, which may have affected the outcomes. Younger age may, for example, have influenced the rehabilitation unit treatment team's expectations for recovery, resulting in delayed discharge and more inpatient and study-related therapy. Younger subjects may have been more comfortable with or more motivated by the robotic technology, promoting a greater tolerance for the additional time spent in training sessions. However, unlike the results observed in our studies with chronic and subacute stroke subjects, neither RA group had significantly greater FMA changes than the CG. Since the mean dose difference between the RA groups was driven by 5 of the 17 Robot-Hi subjects, the overlap in dose between the Robot-Hi and Robot-Lo groups may have masked a group difference. Future studies should be designed to ensure a clear difference between these high- and lowdose groups.

An inverse relationship was found for Ashworth scores, with Robot-Hi subjects demonstrating significant increases in tone at 6 months. Excessive tone can mask recovery of voluntary motor control; therefore, methods have been developed to counter it. The Bobath therapy approach, for example, encourages inhibition of abnormal tone through use of reflex-inhibiting patterns of movement as an important goal [27]. Contrary to what might be expected based on that philosophy, in this study, the group with the greatest increase in tone did as well on recovery of independence in ADLs as the other groups, as reflected in FIM score improvements posttreatment and at 6 months. Since testing site was also a significant factor in the posttreatment FIM score, a stronger focus on restoration of $\mathrm{ADL}$ independence during or after the study intervention cannot be excluded as the cause. Future studies should analyze the means by which tasks are performed during testing of FIM tasks, with special attention to decreased use of compensatory techniques.

Limitations of this study include the relatively small number of subjects in each group and the variation in severity level across subjects. Since brain imaging has become routine for stroke patients, the discovery of small lacunar infarcts during hospital admission is not uncommon. Although such lesions may impact recovery from a major stroke, we did not disqualify subjects just on the basis of a previous stroke as long as no motor or sensory impairments were apparent to the subject before the stroke that precipitated hospital admission. The impact, if any, on the study outcomes is unknown. Subjects with prior major stroke were excluded.

The inconsistent improvement of function in published clinical trials of RA upper-limb rehabilitation helps explain why clinical utilization remains limited in spite of international sales of a growing number of robot-based systems, including the InMotion2 (Interactive Motion 
Technologies Inc; Watertown, Massachusetts), a derivative of the MIT-MANUS, Armeo series (Hocoma Inc, USA; Rockland, Massachusetts; and Hocoma AG; Volketswil, Switzerland), and ReoGo (Motorika USA, Inc; Birmingham, Alabama; and Motorika Medical [Israel] Ltd; Caesarea, Israel). These commercial devices vary greatly in electromechanical design, display features, and operating characteristics. A clear evidence-based rationale for designing, selecting, funding, and integrating robotic systems into rehabilitation clinics remains to be established. Engelhardt first proposed the potential for robots to deliver therapy in 1986 [28]. Controlled clinical trials began in the 1990s, when the emphasis on rehabilitation robot development shifted from assistive applications to RA training for restoration of function following stroke and other disorders $[4,15-16,29]$. The last decade has seen a growing number of published clinical trials and meta-analyses of upperlimb RA rehabilitation therapy. In their recent review of randomized controlled trials, Kwakkel et al. confirmed the potential for robotic systems using a variety of control and movement paradigms to improve proximal upper-limb recovery [10]. However, they also identified a number of issues remaining to be adequately addressed in future clinical trials, including the contribution of training intensity. Although in this study RA training did not result in significant advantages over an equal amount of practice with a therapist, the physical demands of RA training were certainly less. Refinements to this or other approaches may eventually prove to be better than conventional therapy. Robotic systems that facilitate concurrent proximal and distal (including hand) upper-limb exercise are under development, as are additional devices with bimanual capabilities [30]. While grasp is important for efficient performance of many ADLs, proximal joint and limb-segment control expands the volume in which the hand can manipulate objects. Inclusion of hand exercises, coordinated with shoulder and elbow movements in future versions of MIME, may be the key to achieving clinically meaningful functional gains. Also, many combined approaches are being investigated, e.g., RA plus functional electrical stimulation, RA plus virtual reality, and RA plus transcranial direct current stimulation. Some of these approaches may prove more effective than RA training alone.

Although the effect of dose on response was a secondary outcome measure, it has been identified as a very important topic. In 2007, the U.S. Agency for Healthcare Research and Quality supported the development of guide- lines for designing, conducting, and reporting effectiveness reviews comparing two or more therapeutic interventions. Among the secondary constructs that justify increasing the strength of evidence is the presence of a dose-response association [31]. For upper-limb RA therapy, this association has not previously been reported.

When data from all subjects assigned to the RA groups were pooled, regression analysis revealed that patients with lower FMA scores on admission received a higher dose of treatment. This may simply reflect the longer length of stay required for highly impaired patients to meet rehabilitation treatment goals, thus extending eligibility to continue study therapy sessions. At discharge, however, a significant correlation existed between the amount of RA training received and the changes in FMA score. At the 6-month follow-up assessment, following a period with no robot-based movement practice, the correlation had weakened to a trend. An analysis of the intensity of training found even stronger correlations between the average number of hours of therapy per day and FMA changes, both at the end of the active treatment and 6 months later.

It has long been established that improved recovery from stroke can result from additional therapy using conventional methods [32-34]. Increased exposure to novel therapy methods has, however, produced inconsistent results, with reports of positive dose-response effects (learning-based sensorimotor training [35]), no effect of increased dose (neuromuscular electrical stimulation [36]), and an inverse response to higher dose interventions (constraint-induced movement therapy [CIMT] [36]). The latter result raises the possibility of a limit on the amount of RA training that is beneficial. Expansion of neural injury and reduced restoration of function associated with early forced use of a forelimb after unilateral brain injury has been seen in rats [37-40]. Although the applicability of these results to human trials has not been demonstrated, they point out the need to consider intensity, total dose, and timing of training in trials of novel therapeutic methods and devices. In a study on CIMT $9.65 \pm 4.50$ days after stroke, Dromerick et al. assessed a subset of the subjects receiving control therapy, low-intensity CIMT, and high-intensity CIMT with magnetic resonance imaging techniques sensitive to changes following cerebral infarction. Although the sample size was small, no evidence was found for enlargement of the stroke lesion that could have explained the inverse dose-response observed [37]. 
A study by Boake et al. of early CIMT also showed no adverse effect [41].

Masiero et al. conducted a clinical trial of RA therapy delivered using the Neuro-Rehabilitation-roBot (NeReBot) very early $(<1$ week) after stroke at a total dose within the range of that received by experimental subjects in our study [17]. Like MIME, NeReBot facilitates upper-limb movements in three-dimensional space. No adverse effect was seen. Patients who received RA therapy in addition to standard rehabilitation showed greater improvement in FMA and proximal strength in the paretic limb and greater FIM score change compared with controls. Differences exist between Masiero et al.'s study and ours, however. The mean length of stay in acute rehabilitation was 52 to 54 days in their study, more than twice that in ours. Their controls did not receive additional conventional therapy matched to the amount of RA training. Instead, they were exposed to the robot for approximately 25 percent of the RA training time and their exercises were performed with the less-impaired upper limb.

No published human trial has related higher dose of RA training to a decline in outcomes. The ability of the robot to help complete volitional movements may diminish such an effect by not promoting learned nonuse, an adverse effect that was reported in an animal study [42]. This hypothesis warrants investigation in humans. Future studies exploring high-intensity training should also consider the possible existence of an upper limit beyond which harm is done. This is not a new concept. In 1538, Paracelsus, a Swiss-German physician, remarked: "Only the dose makes a thing not to be poison.” Sengai (17501838), a Japanese Zen monk and calligrapher, painted a spoon with the comment "whether for life, whether for death-(it depends on) the right spoon-measure" [43].

In summary, the main result from this study is the demonstration of a trend toward greater upper-limb FMA scores in the RA therapy groups compared with an equal amount of conventional therapy, but only at follow-up and the difference was not significant. This finding contrasts with the more rapid and statistically greater increase in FMA shown in the acute and subacute studies with MIME. The findings suggest that (1) the best use for the capabilities currently designed into MIME is not in the acute recovery period following stroke, (2) with further development the system has the potential to change the trend toward better outcomes into significant improvements, and (3) an upper bound on training dura- tion and intensity in the acute poststroke period remains undefined but appears to exceed what was used here. The greater improvement in FIM score with high dose RA requires further study and confirmation in future clinical trials. Use of MIME as a research intervention was accepted by staff on the acute rehabilitation unit, and they cooperated with scheduling, with the understanding that usual therapy sessions received priority. No adverse effects were reported. The increased tone detected at follow-up in the Robot-Hi subjects was not accompanied by reduced ADL independence, as measured by the FIM scores; this observation also deserves more study. The secondary analysis demonstrated a strong dose-response effect between intensity of training and FMA scores at the completion of training and at follow-up.

\section{CONCLUSIONS}

This study adds to the strength of published research, demonstrating that improved shoulder and elbow function and impairment measures following RA training can be at least equivalent to conventional, labor-intensive methods. Demonstration of the relationship between intensity and motor control adds credibility to RA therapy as the cause of the observed trend in FMA differences. Robotic devices are more likely to be widely accepted if they can be used to increase the intensity and dose beyond what is practicable with therapist assistance and do so in a cost-effective manner. Development of robot-based treatments for upper-limb paresis following stroke is entering a third decade. A clear advantage for recovery of meaningful functional independence with their use, however, has not been conclusively demonstrated. Reviews of randomized clinical trials, including those with MIME, show it is possible to facilitate strength and motor control improvements but still not restore the patient's ability to achieve greater independence. Other methods that do not require a substantial equipment investment can also do this. Remaining questions to be answered include the optimal window for providing therapy with robotic devices, optimal robot hardware and control system design and performance characteristics, patient selection guidelines, the upper limits of intensity and duration beyond which functional penalties may occur, and whether the simultaneous training of proximal and distal upper-limb movements is essential to recovery of meaningful upper-limb use. 


\section{ACKNOWLEDGMENTS}

\section{Author Contributions:}

Study concept and design: C. G. Burgar, P. S. Lum.

Acquisition of data: C. G. Burgar, P. S. Lum, A. M. E. Scremin, S. L. Garber, D. Kenney, P. Shor.

Analysis and interpretation of data: C. G. Burgar, P. S. Lum,

A. M. E. Scremin, S. L. Garber.

Drafting of manuscript: C. G. Burgar, P. S. Lum.

Critical revision of manuscript for important intellectual content:

P. S. Lum, A. M. E. Scremin, S. L. Garber, H. F. M. Van der Loos,

D. Kenney, P. Shor.

Obtained funding; C. G. Burgar, P. S. Lum.

Study supervision: C. G. Burgar.

Administrative support: A. M. E. Scremin.

Study site supervision: A. M. E. Scremin, S. L. Garber, H. F. M. Van der Loos.

Design, installation, and technical support: H. F. M. Van der Loos.

Financial Disclosures: The authors have declared that no competing interests exist.

Funding/Support: This material was based on work supported by the VA Rehabilitation Research and Development Service (grant B2695I). Additional Contributions: We thank Carol Leach, PT; Zaven Ghezelashouri, MPH; and Henry Lew, MD, PhD, for their valuable assistance with carrying out the study and subject recruitment. During planning and initiation of the clinical trial, Drs. Burgar, Lum, and Van der Loos were affiliated with the VA Palo Alto Health Care System, Palo Alto, California, and the Department of Functional Restoration, Stanford University, Stanford, California. Since study completion, Ms. Garber ended her affiliation with the Michael E. DeBakey VA Medical Center. Dr. Van der Loos has relocated to the Department of Mechanical Engineering, University of British Columbia, Vancouver, Canada. Ms. Kenney is now also affiliated with the Department of Orthopaedic Surgery, Stanford University. Ms. Shor is currently in private practice in Menlo Park, California.

Institutional Review: Approval for participation in the trials was obtained from each site's institutional review board and research and development committee and, for data analysis, at the Central Texas Veterans Health Care System (Temple, Texas).

Participant Follow-Up: The authors do not plan to inform participants of the publication of this study.

\section{REFERENCES}

1. National Center for Health Statistics. Health, United States, 2009: With special feature on medical technology. Hyattsville (MD): U.S. Department of Health and Human Services, Centers for Disease Control and Prevention, National Center for Health Statistics; 2010.

2. Centers for Disease Control and Prevention (CDC). Prevalence of disabilities and associated health conditions among adults-United States, 1999. MMWR Morb Mortal Wkly Rep. 2001;50(7):120-25. [PMID: 11393491$]$
3. Writing Group Members, Lloyd-Jones D, Adams RJ, Brown TM, Carnethon M, Dai S, De Simone G, Ferguson TB, Ford E, Furie K, Gillespie C, Go A, Greenlund K, Haase N, Hailpern S, Ho PM, Howard V, Kissela B, Kittner S, Lackland D, Lisabeth L, Marelli A, McDermott MM, Meigs J, Mozaffarian D, Mussolino M, Nichol G, Roger VL, Rosamond W, Sacco R, Sorlie P, Roger VL, Thom T, Wasserthiel-Smoller S, Wong ND, Wylie-Rosett J; American Heart Association Statistics Committee and Stroke Statistics Subcommittee. Heart disease and stroke statistics-2010 update: A report from the American Heart Association. Circulation. 2010;121(7):e46-e216. [PMID: 20019324] DOI:10.1161/CIRCULATIONAHA.109.192667

4. Burgar CG, Lum PS, Shor PC, Van der Loos M. Development of robots for rehabilitation therapy: The Palo Alto VA/Stanford Experience. J Rehabil Res Dev. 2000;37(6): 663-73. [PMID: 11321002]

5. Lum PS, Burgar CG, Shor PC, Majmundar M, Van der Loos M. Robot-assisted movement training compared with conventional therapy techniques for the rehabilitation of upper-limb motor function after stroke. Arch Phys Med Rehabil. 2002;83(7):952-59. [PMID: 12098155]

6. Lum PS, Burgar CG, Shor PC. Evidence for improved muscle activation patterns after retraining of reaching movements with the MIME robotic system in subjects with post-stroke hemiparesis. IEEE Trans Neural Syst Rehabil Eng. 2004;12(2):186-94. [PMID: 15218933] DOI:10.1109/TNSRE.2004.827225

7. Lum PS, Burgar CG, Van der Loos M, Shor PC, Majmundar M, Yap R. The MIME robotic system for upper-limb neurorehabilitation: Results from a clinical trial in subacute stroke. Proceedings of the 9th International Conference on Rehabilitation Robotics; 2005 Jun 28-Jul 1; Chicago, IL. Los Alamitos (CA): IEEE; 2005. p. 511-14. DOI:10.1109/ICORR.2005.1501153

8. Lum PS, Burgar CG, Van der Loos M, Shor PC, Majmundar M, Yap R. MIME robotic device for upper-limb neurorehabilitation in subacute stroke subjects: A follow-up study. J Rehabil Res Dev. 2006;43(5):631-42. [PMID: 17123204] DOI:10.1682/JRRD.2005.02.0044

9. Fugl-Meyer AR, Jääskö L, Leyman I, Olsson S, Steglind S. The post-stroke hemiplegic patient. 1. A method for evaluation of physical performance. Scand J Rehabil Med. 1975; 7(1):13-31. [PMID: 1135616]

10. Kwakkel G, Kollen BJ, Krebs HI. Effects of robot-assisted therapy on upper limb recovery after stroke: A systematic review. Neurorehabil Neural Repair. 2008;22(2):111-21.

[PMID: 17876068] DOI:10.1177/1545968307305457

11. Prange GB, Jannink MJ, Groothuis-Oudshoorn CG, Hermens HJ, IJzerman MJ. Systematic review of the effect of robot-aided therapy on recovery of the hemiparetic arm 
after stroke. J Rehabil Res Dev. 2006;43(2):171-84.

[PMID: 16847784$]$

DOI:10.1682/JRRD.2005.04.0076

12. Mehrholz J, Platz T, Kugler J, Pohl M. Electromechanical and robot-assisted arm training for improving arm function and activities of daily living after stroke. Cochrane Database Syst Rev. 2008;8(4):CD006876. [PMID: 18843735]

13. Lo AC, Guarino PD, Richards LG, Haselkorn JK, Wittenberg GF, Federman DG, Ringer RJ, Wagner TH, Krebs HI, Volpe BT, Bever CT Jr, Bravata DM, Duncan PW, Corn BH, Maffucci AD, Nadeau SE, Conroy SS, Powell JM, Huang GD, Peduzzi P. Robot-assisted therapy for long-term upper-limb impairment after stroke. N Engl J Med. 2010; 362(19):1772-83. [PMID: 20400552] DOI:10.1056/NEJMoa0911341

14. Hesse S, Werner C, Pohl M, Rueckriem S, Mehrholz J, Lingnau ML. Computerized arm training improves the motor control of the severely affected arm after stroke: A single-blinded randomized trial in two centers. Stroke. 2005; 36(9):1960-66. [PMID: 16109908] DOI:10.1161/01.STR.0000177865.37334.ce

15. Aisen ML, Krebs HI, Hogan N, McDowell F, Volpe BT. The effect of robot-assisted therapy and rehabilitative training on motor recovery following stroke. Arch Neurol. 1997; 54(4):443-46. [PMID: 9109746]

16. Volpe BT, Krebs HI, Hogan N, Edelsteinn L, Diels CM, Aisen ML. Robot training enhanced motor outcome in patients with stroke maintained over 3 years. Neurology. 1999;53(8):1874-76. [PMID: 10563646]

17. Masiero S, Celia A, Rosati G, Armani M. Robotic-assisted rehabilitation of the upper limb after acute stroke. Arch Phys Med Rehabil. 2007;88(2):142-49. [PMID: 17270510] DOI:10.1016/j.apmr.2006.10.032

18. Hamilton BB, Granger CV, Sherwin FS, Zielezny M, Tashman JS. A uniform national data system for medical rehabilitation. In: Fuhrer MJ, editor. Rehabilitation outcomes: Analysis and measurement. Baltimore (MD): Paul H. Brookes; 1987. p. 137-47.

19. Bleecker ML, Bolla-Wilson K, Kawas C, Agnew J. Agespecific norms for the Mini-Mental State Exam. Neurology. 1988;38(10):1565-68. [PMID: 3419600]

20. Duncan PW, Propst M, Nelson SG. Reliability of the FuglMeyer assessment of sensorimotor recovery following cerebrovascular accident. Phys Ther. 1983;63(10):1606-10. [PMID: 6622535]

21. Gladstone DJ, Danells CJ, Black SE. The Fugl-Meyer assessment of motor recovery after stroke: A critical review of its measurement properties. Neurorehabil Neural Repair. 2002;16(3):232-40. [PMID: 12234086] DOI:10.1177/154596802401105171
22. Hamilton BB, Laughlin JA, Granger CV, Kayton RM. Interrater agreement of the seven-level Functional Independence Measure (FIM). Arch Phys Med Rehabil. 1991;72:790.

23. Ashworth B. Preliminary trial of carisoprodol in multiple sclerosis. Practitioner. 1964;192:540-42. [PMID: 14143329]

24. Bohannon RW, Smith MB. Interrater reliability of a modified Ashworth scale of muscle spasticity. Phys Ther. 1987; 67(2):206-7. [PMID: 3809245]

25. Wolf SL, Catlin PA, Ellis M, Archer AL, Morgan B, Piacentino A. Assessing Wolf Motor Function Test as outcome measure for research in patients after stroke. Stroke. 2001; 32(7):1635-39. [PMID: 11441212]

26. Morris DM, Uswatte G, Crago JE, Cook EW 3rd, Taub E. The reliability of the Wolf Motor Function Test for assessing upper extremity function after stroke. Arch Phys Med Rehabil. 2001;82(6):750-55. [PMID: 11387578] DOI:10.1053/apmr.2001.23183

27. Bobath B. Adult hemiplegia: Evaluation and treatment. 2nd ed. London (England): Heinemann Medical; 1978.

28. Engelhardt KG. Current status and future prospects for robotic technology in health care delivery. Cent Nerv Syst Trauma. 1986;3(1):111-26. [PMID: 3731287]

29. Volpe BT, Krebs HI, Hogan N, Edelstein L, Diels C, Aisen M. A novel approach to stroke rehabilitation: Robot-aided sensorimotor stimulation. Neurology. 2000;54(10):1938-44. [PMID: 10822433]

30. Brewer BR, McDowell SK, Worthen-Chaudhari LC. Poststroke upper extremity rehabilitation: A review of robotic systems and clinical results. Top Stroke Rehabil. 2007; 14(6):22-44. [PMID: 18174114$]$ DOI:10.1310/tsr1406-22

31. Owens DK, Lohr KN, Atkins D, Treadwell JR, Reston JT, Bass EB, Chang S, Helfand M. Grading the strength of a body of evidence when comparing medical interventions [Internet]. Rockville (MD): Agency for Healthcare Research and Quality; 2009. Available from: http://effectivehealthcare.ahrq.gov/ehc/products/122/328/ 20090805 grading.pdf.

32. Smith ME, Garraway WM, Smith DL, Akhtar AJ. Therapy impact on functional outcome in a controlled trial of stroke rehabilitation. Arch Phys Med Rehabil. 1982;63(1):21-24. [PMID: 7034669]

33. Sunderland A, Tinson DJ, Bradley EL, Fletcher D, Langton Hewer R, Wade DT. Enhanced physical therapy improves recovery of arm function after stroke. A randomised controlled trial. J Neurol Neurosurg Psychiatry. 1992;55(7): 530-35. [PMID: 1640226]

DOI:10.1136/jnnp.55.7.530

34. Dam M, Tonin P, Casson S, Ermani M, Pizzolato G, Iaia V, Battistin L. The effects of long-term rehabilitation therapy on poststroke hemiplegic patients. Stroke. 1993;24(8): 1186-91. [PMID: 8342195] 
35. Byl NN, Pitsch EA, Abrams GM. Functional outcomes can vary by dose: Learning-based sensorimotor training for patients stable poststroke. Neurorehabil Neural Repair. 2008; 22(5):494-504. [PMID: 18780884]

DOI:10.1177/1545968308317431

36. Hsu SS, Hu MH, Wang YH, Yip PK, Chiu JW, Hsieh CL. Dose-response relation between neuromuscular electrical stimulation and upper-extremity function in patients with stroke. Stroke. 2010;41(4):821-24. [PMID: 20203321]

DOI:10.1161/STROKEAHA.109.574160

37. Dromerick AW, Lang CE, Birkenmeier RL, Wagner JM, Miller JP, Videen TO, Powers WJ, Wolf SL, Edwards DF. Very Early Constraint-Induced Movement during Stroke Rehabilitation (VECTORS): A single-center RCT. Neurology. 2009;73(3):195-201. [PMID: 19458319]

DOI:10.1212/WNL.0b013e3181ab2b27

38. Humm JL, Kozlowski DA, James DC, Gotts JE, Schallert T. Use-dependent exacerbation of brain damage occurs during an early post-lesion vulnerable period. Brain Res. 1998;783(2):286-92. [PMID: 9507166] DOI:10.1016/S0006-8993(97)01356-5

39. Humm JL, Kozlowski DA, Bland ST, James DC, Schallert T. Use-dependent exaggeration of brain injury: Is glutamate involved? Exp Neurol. 1999;157(2):349-58.

[PMID: 10364446]

DOI:10.1006/exnr.1999.7061

40. Bland ST, Schallert T, Strong R, Aronowski J, Grotta JC, Feeney DM. Early exclusive use of the affected forelimb after moderate transient focal ischemia in rats: Functional and anatomic outcome. Stroke. 2000;31(5):1144-52. [PMID: 10797179]
41. Boake C, Noser EA, Ro T, Baraniuk S, Gaber M, Johnson R, Salmeron ET, Tran TM, Lai JM, Taub E, Moye LA, Grotta JC, Levin HS. Constraint-induced movement therapy during early stroke rehabilitation. Neurorehabil Neural Repair. 2007;21(1):14-24. [PMID: 17172550] DOI:10.1177/1545968306291858

42. Erickson CA, Gharbawie OA, Whishaw IQ. Attemptdependent decrease in skilled reaching characterizes the acute postsurgical period following a forelimb motor cortex lesion: An experimental demonstration of learned nonuse in the rat. Behav Brain Res. 2007;179(2):208-18. [PMID: 17346809] DOI:10.1016/j.bbr.2007.02.004

43. Stumpf WE. The dose makes the medicine. Drug Discov Today. 2006;11(11-12):550-55.

Submitted for publication April 8, 2010. Accepted in revised form November 9, 2010.

This article and any supplementary material should be cited as follows:

Burgar CG, Lum PS, Scremin AME, Garber SL, Van der Loos HFM, Kenney D, Shor P. Robot-assisted upperlimb therapy in acute rehabilitation setting following stroke: Department of Veterans Affairs multisite clinical trial. J Rehabil Res Dev. 2011;48(4):445-58.

DOI:10.1682/JRRD.2010.04.0062

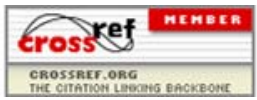

